\title{
E-Learning 3-D - welches Potenzial haben virtuelle 3-D-Umgebungen für das Lernen mit neuen Medien?
}

Henning Pätzold

\begin{abstract}
Die gegenwärtige Debatte um virtuelle 3-D-Umgebungen fordert nicht zuletzt die Medienpädagogik heraus. Welche didaktischen Potenziale haben solche Umgebungen und welche pädagogischen Szenarien werden hier bereits verwirklicht? Vor dem Hintergrund einer Darstellung aktuell diskutierter 3-D-Umgebungen, pädagogischer Projekte und Konzepten multimedialen Lernens sowie der handlungsorientierten Mediendidaktik wird diesen Fragen nachgegangen. Den Abschluss bilden eine Abschätzung des mediendidaktischen Potenzials und ein Ausblick.
\end{abstract}

\section{Einführung}

Weltweit vernetzte 3-D-Welten spielen schon seit längerem in Mediendebatten eine Rolle, waren aber bislang durch technische Möglichkeiten stark begrenzt. Während sich allerdings die technische Phantasie vor allem auf die Entwicklung verbesserter Ein-Ausgabe-Schnittstellen (Datenhandschuh, 3-D-Brille usw.) konzentrierte, spielte sich parallel dazu durch die schnelle Entwicklung der verfügbaren Netzbandbreiten und der «traditionellen» Hardware (Rechenleistung, Speicher, Grafik) eine kleine Revolution ab, die es möglich macht, dass inzwischen MMPORGs ${ }^{1}$ mit Zehntausenden gleichzeitig aktiven Nutzern bei hoher grafischer Qualität technisch beherrschbar sind. Insbesondere erlaubt die Verfügbarkeit hohe Bandbreiten und damit die Realisierung aufwändiger grafischer Szenarien am Bildschirm. Interessanterweise tritt mit der Verbesserung der Interaktionsmöglichkeiten die Bedeutung eines klaren Spielsettings in den Hintergrund - pointiert gesagt lässt die Notwendigkeit, Drachen zu töten und Jungfrauen zu befreien nach, wenn man auch befriedigende Gespräche mit anderen Usern führen kann.

Diese Entwicklungen haben natürlich auch vielfältige medienpädagogische Implikationen: Nicht nur die Frage, ob der Aufenthalt in anregenden virtuellen Räumen vielleicht negative Auswirkungen auf die Entwicklung realen Sozial- und Lernverhaltens hat, wird diskutiert, sondern im Sinne einer handlungsorientierten Medienpädagogik (vgl. Tulodziecki und Herzig 2004: 185ff, Vollbrecht 2001: 51f) geht es auch darum, ob und wie das Lernen als allgemeinmenschlicher Vorgang in virtuellen Welten einen angemessenen Raum finden kann - oder ob 
diese Räume zumindest eine didaktische Erweiterung bestehender Lehr- und Lernmöglichkeiten darstellen. Der zweiten Fragerichtung soll im Folgenden genauer nachgegangen werden. Hierzu erfolgt zuerst ein Überblick über die Entwicklung und Möglichkeiten einschlägiger virtueller Multi-User-Welten. Im Anschluss daran werden konkrete medienpädagogische Szenarien skizziert, die in solchen Welten umgesetzt werden, und schliesslich erfolgt auf dieser Grundlage eine Bewertung der gegenwärtigen Möglichkeiten und Grenzen und des medienpädagogischen Potenzials.

\section{Virtuelle 3-D-Umgebungen - ein Überlick}

Wie in anderen Bereichen technologischer Entwicklung auch ist die Terminologie bei den virtuellen 3-D-Umgebungen nicht einheitlich. Der Begriff MMPORG deckt wesentliche Bereiche ab, bezieht sich aber ausschliesslich auf Rollenspielwelten, wohingegen neuere Entwicklungen sich von Spielszenarien immer weiter entfernen. Bezeichnungen wie virtuelle Welt, virtual reality usw. sind eng an die Diskussion um eine möglichst vollständige Abbildung von Sinneseindrücken durch Technik geknüpft (vgl. Bühl 2000: 120)². Die gegenwärtige Entwicklung konzentriert sich jedoch - wie schon bei den MUDs ${ }^{3}$ der 1980er und 1990er Jahre - auf grosse Zielgruppen, die erreicht werden, indem weit gehend auf eine technische Standardausstattung gesetzt wird. Eine wichtige Bedingung gegenüber früheren Simulationen besteht dabei in der Implementierung einer möglichst überzeugenden dreidimensionalen Darstellung der Szenarien und damit komplexerer Bewegungsmöglichkeiten. Der hier verwendete Begriff virtuelle 3D-Umgebungen soll deutlich machen, dass

- die Umgebung eine anspruchsvolle dreidimensionale Darstellung aufweist, in der prinzipiell «Bewegungen» in allen Raumrichtungen möglich sind,

- es sich um eine virtuelle Umgebung in dem Sinne handelt, dass sie keiner realen Welt entspricht, aber Aspekte der realen Welt abbildet und eine anspruchsvolle soziale Interaktion zwischen den Akteuren, vielleicht auch zwischen Akteur und Maschine erlaubt - Peterson spricht in diesem Zusammenhang von der «E-Condition» (Peterson 2003), Turkle von Maschinen und Programmen als «relational artifacts» (Turkle 2006: 18) - und

- diese Umgebungen zumindest nicht hauptsächlich zur Realisierung einer vorstrukturieren Spielhandlung eingesetzt werden ${ }^{4}$.

Ein wesentliches Merkmal solcher 3-D-Umgebungen besteht darin, dass die Schnittstellen zu sozialen Phänomenen der realen Welt ${ }^{5}$ relativ offen sind. Insbesondere ist ein teilweiser Geldaustausch zwischen virtueller und realer Welt möglich. Die Plattform Second Life, auf die unten eingegangen wird, war dabei nicht der erste Anbieter, der es erlaubt, mit realem Geld virtuelle Ausstattung zu kaufen - bei verschiedenen MMPORGs (z. B. World of Warcraft, Final Fantasy, 
Entropia Universe usw.) gibt es einen realen Handel mit virtuellen Artefakten. Bei second life wird aber erstmalig die Möglichkeit diskutiert, in der virtuellen Welt Geld zu verdienen, das in der Realen Welt zum Lebensunterhalt wird (vgl. Turkle 2006: 6). Mit der Konvertibilität virtuellen in echtes Geld, wie sie bei einigen 3-D-Umgebungen möglich ist, ist eine deutlich «allgemeinere» Verknüpfung zwischen realer und virtueller Welt verbunden - prinzipiell kann sich jeder Akteur in der virtuellen Welt für alle Arten von Waren und Dienstleistungen bezahlen lassen, die dort verfügbar sind - und das erworbene Geld in reales Geld umtauschen. ${ }^{6}$ Aber die Konvertibilität zwischen virtueller und realer Welt scheint sich nicht auf eine Währung zu beschränken. In dem Umfang, in dem virtuelle Umgebungen sozialen Austausch erlauben, besteht auch die Möglichkeit, die Befriedigung sozialer Bedürfnisse z. B. nach Anerkennung zu erreichen, wobei hierfür in beiden Sphären signifikant unterschiedliche Voraussetzungen gelten. Ein nach allgemeinen Ansprüchen «schöner» Körper gehört etwa in Second Life zur «Standardausstattung» (vgl. Turkle 2006: 7). Er bildet die visuelle Basis für Interaktionen mit anderen Teilnehmern, lässt sich aber durch Geschick und/oder den Einsatz virtuellen Kapitals auch noch weiter entwickeln.

Alle virtuellen 3-D-Umgebungen bieten daneben natürlich die Möglichkeit, schriftsprachlich oder sogar per Audioübertragung mit anderen Akteuren in Kontakt zu treten. Damit schliessen sie an verschiedene Formen der textbasierten vernetzen Interaktionsumgebungen an. Zum einen wären die MUDs zu nennen, die ursprünglich Spielwelten darstellten, mit der Zeit aber ihrerseits zum Teil zu Interaktionsumgebungen ohne spezifische Spielhandlung wurden. Zum anderen sind hier die zahlreichen Chatsysteme zu nennen, deren Urform der Internet Relay Chat (IRC) darstellt.

Die Entwicklung virtueller 3-D-Umgebungen hat also mit Spielen begonnen, zunehmend ist aber eine "Emanzipation» von diesem Anwendungsbereich zu beobachten. Wenngleich auch aktuelle Produkte zumindest implizit bestimmte Zielgruppen zu bedienen scheinen, bieten sie eine deutlich grössere Bandbreite potenzieller Interaktionen. Im Folgenden wird ein Überblick über aktuelle 3-D-Umgebungen gegeben.

Second Life: Das «Second Life» der Firma Linden Labs stellt in der westlichen Welt die zurzeit bedeutendste virtuelle 3-D-Welt dar und ist gegenwärtig Gegenstand einer umfangreichen Diskussion (z. B. Hamann/Uehlecke 2007). Ein Blick auf die Historie der Suchanfragen zu Second Life (vgl. Abbildung 1) zeigt das steile Ansteigen des Interesses an diesem Produkt, dass bereits 2003 online gegangen ist. Second Life ist eine virtuelle 3-D-Umgebung, die den Akteuren eine Navigation in einer sehr aufwändig gestalteten Umgebung erlaubt, die weitestgehend von den Nutzerinnen und Nutzern selbst erschaffen wird. Sie finden hier die Möglichkeit, über entsprechende Software eigene Artefak- 


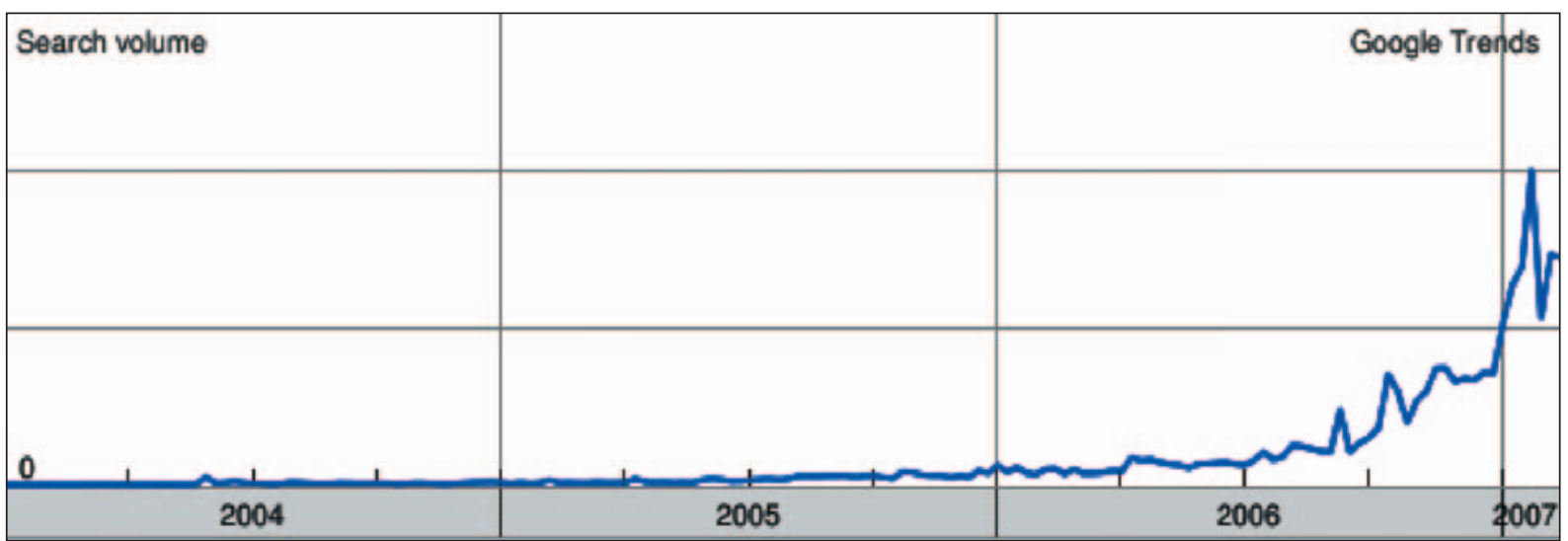

Abbildung 1: Trendkurve zum Suchbegriff «Second Life» bei Google (www.google.com/trends, Abfrage vom 18.7.2007)

te zu konstruieren und in der virtuellen Umgebung einzusetzen. Ebenso lassen sich Gesten und anderes für die virtuellen Figuren («Avatare» - ein aus dem Sanskrit entlehnter Begriff, der eigentlich einen auf die Welt herabgestiegenen Gott bezeichnet) «herstellen» und - teilweise kostenpflichtig - in die virtuelle Welt einbinden. Die Interaktionsmöglichkeiten in Second Life sind grundsätzlich wenig beschränkt und es gibt ausdrücklich keine Spielidee oder Ähnliches. Tatsächlich bilden sich bestimmte Handlungsbereiche aus, die zum Teil anderen kommerzialisierten Räumen des Internet recht ähnlich sind. So gibt es auch hier Pornografie und Glücksspiel, daneben eine Reihe von Firmenauftritten, etwa im Lifestylebereich, aber auch Angebote von Bildungseinrichtungen.

Moove: Moove ist eine alternative 3-D-Interaktionsumgebung, die auf einem etwas anderen technischen Prinzip basiert. Während Second Life über ein umfangreiches System zentraler Server gesteuert wird, wird bei Moove ein dezentrales Netzwerk von so genannten «Häusern» gebildet (sie bilden, mit Gegenständen individuell eingerichtet, die Metapher des Interaktionsraumes). Jeder Benutzer hat ein solches Haus, in das er andere Nutzer einladen oder von dem aus er andere besuchen kann. Damit gibt es zwar eine Reihe von Interaktionsmöglichkeiten, aber in der Umgebung selbst keine "Öffentlichkeit», was mit ein Grund dafür sein dürfte, dass Moove besonders grosse Ähnlichkeiten mit chatbasierten Kontaktbörsen aufweist und sich für pädagogisch fortschrittliche Settings nur begrenzt eignet. Hier wie dort ist die zum System gehörige Website das Hauptforum für den öffentlichen Austausch.

Active Worlds: Mit der wachsenden Popularität von Second Life bleibt es nicht aus, dass Konkurrenten ähnliche Konzepte umsetzen. Active Worlds bietet ebenso wie Second Life eine 3-D-Umgebung, die ohne Spielidee oder Ähnliches aufwartet und damit zu wenig vorstrukturierter Interaktion bereit steht. 
Auch hier können Interessierte eigenes «Land» erwerben und gestalten. Die Unterschiede zu Second Life bestehen vor allem in der technischen Umsetzung und einem etwas anderen Finanzierungskonzept sowie den bisher wesentlich geringeren Benutzerzahlen. Ausserdem bietet Active Worlds die Möglichkeit, eigene «Welten» zu kreieren, die die Software des Anbieters nutzen, aber nicht an die "Hauptwelt» angeschlossen sind und von dieser aus nicht betreten werden können. Active Worlds wurde ursprünglich in der Absicht entwickelt, eine 3-D-Version des Browsings zu etablieren. Analog hierzu können diese eigenen Welten als «Intranets» verstanden werden.

Sloodle: Sloodle stellt selbst keine virtuelle 3-D-Umgebung dar, sondern den Versuch, die Interaktionsmöglichkeiten solcher Umgebungen mit den didaktischen Arrangements des Learning Management Systems Moodle zu verbinden (vgl. www.sloodle.com [18.7.07]). Bisher ist es möglich, z. B. Texte oder Bildschirmschnappschüsse direkt aus Second Life in entsprechende Moodle-Werkzeuge, z. B. Blogs, zu senden. Damit erlaubt ein solches Interface, die Interaktion im Rahmen von Lehr-Lern-Prozessen dynamisch auf die beiden Ebenen der 3-D-Umgebung und des Learning-Management-Systems zu verteilen.

\section{Pädagogische Möglichkeiten: Projekte und Erfahrungen}

Die pädagogischen Möglichkeiten, die virtuelle 3-D-Umgebungen anbieten, reichen weit: Sie vereinfachen die Kommunikation zwischen den Akteuren und führen sie näher an «natürliche» Interaktionsformen heran. Daneben bieten sie durch die Raummetapher umfangreiche und oft intuitive Navigationsmöglichkeiten. Schliesslich eignen sie sich zum Aufbau von Simulationen, weil einerseits ein räumlich-visueller Kontext besteht, der der realen Welt nachempfunden ist, andererseits keine eigentlichen Naturgesetze gelten. Entsprechend - und auch weil der Sektor Erziehung und Bildung in der Informationsgesellschaft einen bedeutenden Markt darstellt - gab es bereits früh und mitunter mit Förderung der beteiligten Unternehmen pädagogische Anwendungen in solchen Welten. Hier einen Überblick zu gewinnen ist nicht einfach, weil die Entwicklung sehr dynamisch ist. Für Second Life sind zentrale Projekte jedoch im Rahmen der «Second Life Community Convention» dokumentiert (Livingstone, Kemp 2006), in Active Worlds werden pädagogische Projekte durch das Unternehmenseigene AWEDU (Active Worlds Educational Universe, edu.activeworlds.com, 18.7.07) begleitet.

Für einen systematischen Überblick sind bisher nicht ausreichend viele Projekte etabliert und vor allem angemessen dokumentiert. Bei einem ersten Test am 27.3.07 war keine der bei AWEDU gelisteten Welten für Besucher erreichbar. In einem zweiten Test (18.7.07) waren einige Welten erreichbar', in keiner gab es jedoch andere Besucher. Bei Livingstone und Kemp (2006) sind einige Bei- 
spiele genauer beschrieben, etwa das "'Splo», eine Art naturwissenschaftliches Museum mit Hörsälen, Exponaten und natürlich der Möglichkeit, mit anderen interessierten Besuchern Kontakt aufzunehmen (vgl. Doherty, Rothfarb, Barker 2006). Neben dem 'Splo gibt es eine Vielzahl weiterer Museen und pädagogisch orientierter Ausstellungen unterschiedlicher Qualität.

Eine andere Art der pädagogischen Nutzung stellen Projekte dar, in denen virtuelle 3-D-Umgebungen in institutionalisierte Bildungsprozesse integriert werden, zum Beispiel an der Rheinischen Fachhochschule Köln, die mit einem solchen Angebot «nebenbei» eine PR-Resonanz mit geschätzten 20 Mio. Kontakten erzielt hat (vgl. http://rfh-cologne.de/de/aktuelles/meldungen/20070305. php [27.3.2007]). Wie bei der Integration solcher Umgebungen mit «konventionellen» Learning-Management-Systemen wird die 3-D-Welt hier zum Echtzeit-Interaktionsraum, während andere Lernaktivitäten in einer konventionellen Lernumgebung verbleiben.

Überhaupt steht gegenwärtig noch häufig die PR im Mittelpunkt, wenn Bildungsanbieter Informations- und Serviceeinrichtungen in virtuellen 3-D-Umgebungen etablieren, um etwa interessierte Studienanfänger anzusprechen. Naturgemäss ist diese Tendenz umso stärker, je mehr solche Einrichtungen in einem von Konkurrenzökonomie geprägten Umfeld agieren, wie es beispielsweise bei Spitzenuniversitäten der USA der Fall ist. Pädagogische Einrichtungen gehen dann wie andere Unternehmen vor, indem sie eine attraktive Zielgruppe dort ansprechen, wo sie erreichbar ist und die Einrichtung selbst durch ihr Auftreten Modernität und Qualität demonstrieren kann. Ein weiteres Beispiel, das sowohl von Unternehmen als auch von pädagogisch orientierten Einrichtungen genutzt wird, ist die Entwicklung von Prototypen. Ebenso wie ein Modehersteller die Akzeptanz neuer Designs in der virtuellen Umgebung testen kann, lassen sich, entsprechende Bedienungskenntnisse vorausgesetzt, in virtuellen Umgebungen Prototypen realer Lernmedien herstellen, die dann auch ausserhalb dieser Umgebungen produziert und verwendet werden können (für ein Beispiel eines Museumsexponats vgl. Doherty, Rothfarb, Barker 2006: 22).

Insofern bleiben die bisher realisierten Projekte hinter den Möglichkeiten virtueller 3-D-Umgebungen zumeist noch zurück. Sie präsentieren grossteils konventionelle Formate (Museen, Exponate, Simulationen), die sie allerdings um die Schaffung komplexer Kommunikationsräumen erweitern. So geht es bei einem virtuellen Museum nicht nur darum, Exponate zu zeigen. Vielmehr begegnet man beim virtuellen Museumsbesuch anderen Figuren, mit denen man sich über das Gezeigte direkt und zwanglos austauschen kann. Insbesondere angesichts des hohen Bedürfnisses, Erfahrungen, Eindrücke und Gefühle nicht nur alleine zu reflektieren, sondern unmittelbar (mit)zuteilen (vgl. Turkle 2006), scheint hier in der Tat ein erhebliches Potenzial für kooperatives, soziales Lernen zu liegen. In dieser Perspektive stellt die Förderung virtueller Gemeinschaften des 
Lernens durch die Schaffung gemeinsamer, interaktionsfreundlicher Lernräume eine weitere pädagogische Option dar.

\section{Beurteilung}

Eine pädagogische Beurteilung der Möglichkeiten und Grenzen virtueller 3-DUmgebungen kann beim gegenwärtigen Stand der Entwicklung (und bei ihrer Geschwindigkeit) nicht viel mehr als eine Momentaufnahme sein, die vielleicht einige Entwicklungen der nahen Zukunft antizipiert. Eine erste Orientierung bietet dabei die Kriterien multimedialer Lernumgebungen, die von Bloh und Lehmann aufgeführt werden:

- Multimedialität, also die Verknüpfung verschiedener medialer Darstellungsformung (Bewegtbild, Audio, Text),

- Interaktivität/Adaptivität, womit hier die Interaktion und Veränderbarkeit der Systemumgebung gemeint ist, in «klassischen» Lernplattformen beispielsweise die Gestaltung der Einstiegsseite und

- Vernetzungsaspekte, die die Möglichkeit der synchronen und asynchronen Kommunikation mit anderen Benutzern betreffen (vgl. Bloh, Lehmann 2002: 14, vgl. auch Meschenmoser 2002: 75 ff.).

Mit Blick auf die besonderen Eigenschaften von virtuellen 3-D-Umgebungen und den Anspruch einer handlungsorientierten Mediendidaktik (vgl. auch Tulodziecki und Herzig 2004: 194) sind diese Punkte zu ergänzen um

- soziale Räume, also Interaktionsräume, in denen die Möglichkeit für die Entstehung und Aufrechterhaltung von Interaktionsbeziehungen zwischen den Nutzenden gegeben ist,

- Gemeinschaft, also das tatsächliche und mehr oder weniger dauerhafte Entstehen solcher Beziehungen und

- Integration, womit hier die Möglichkeit gemeint ist, die in einer solchen Umgebung gemachten Erfahrungen der Gemeinschaft und des Lernens auch in das Denken, Fühlen und Handeln im realen Leben zu integrieren.

\section{Multimedialität}

In technischer Hinsicht ist die Multimedialität aktueller 3-D-Umgebungen geradezu ein Massstab für den Stand des technisch Möglichen, soweit es um Software geht, die auf Standardcomputern lauffähig ist. Bei Second Life werden allerdings sogar besondere Anforderungen an die Hardware gestellt. ${ }^{8}$ Unter den Bedingungen eines modernen Rechners mit eher überdurchschnittlicher Hardwareausstattung lassen sich in diesen Umgebungen alle digitalen medialen Möglichkeiten integrieren, sodass sich der Benutzer in einer grafisch recht überzeugenden Umgebung bewegen und dabei sowohl Audioinformationen aus dieser Umgebung empfangen als auch spezielle Streams mit Audio- und/oder 
Videoinformationen empfangen kann. Die Navigation ist dabei nicht auf das herumwandern oder -fliegen begrenzt, sondern es besteht, analog zum Browsing im World Wide Web, auch die Möglichkeit, unmittelbar von einem Ort zu einem anderen zu wechseln, sodass Hypermediastrukturen mit potenziell weit reichendem Einfluss auf Erkenntnisprozesse entstehen (vgl. Forrai 2003). Die technischen Studien von Sloodle zeigen dabei ein erstaunliches Mass an Integration (vgl. Abbildung 2), wenngleich das dargestellte Arrangement didaktisch einen recht konventionellen Eindruck macht und wiederum zeigt, dass das Modell der Ausstellung besonders gut zu den Bedingungen virtueller 3-D-Umgebungen zu passen scheint.

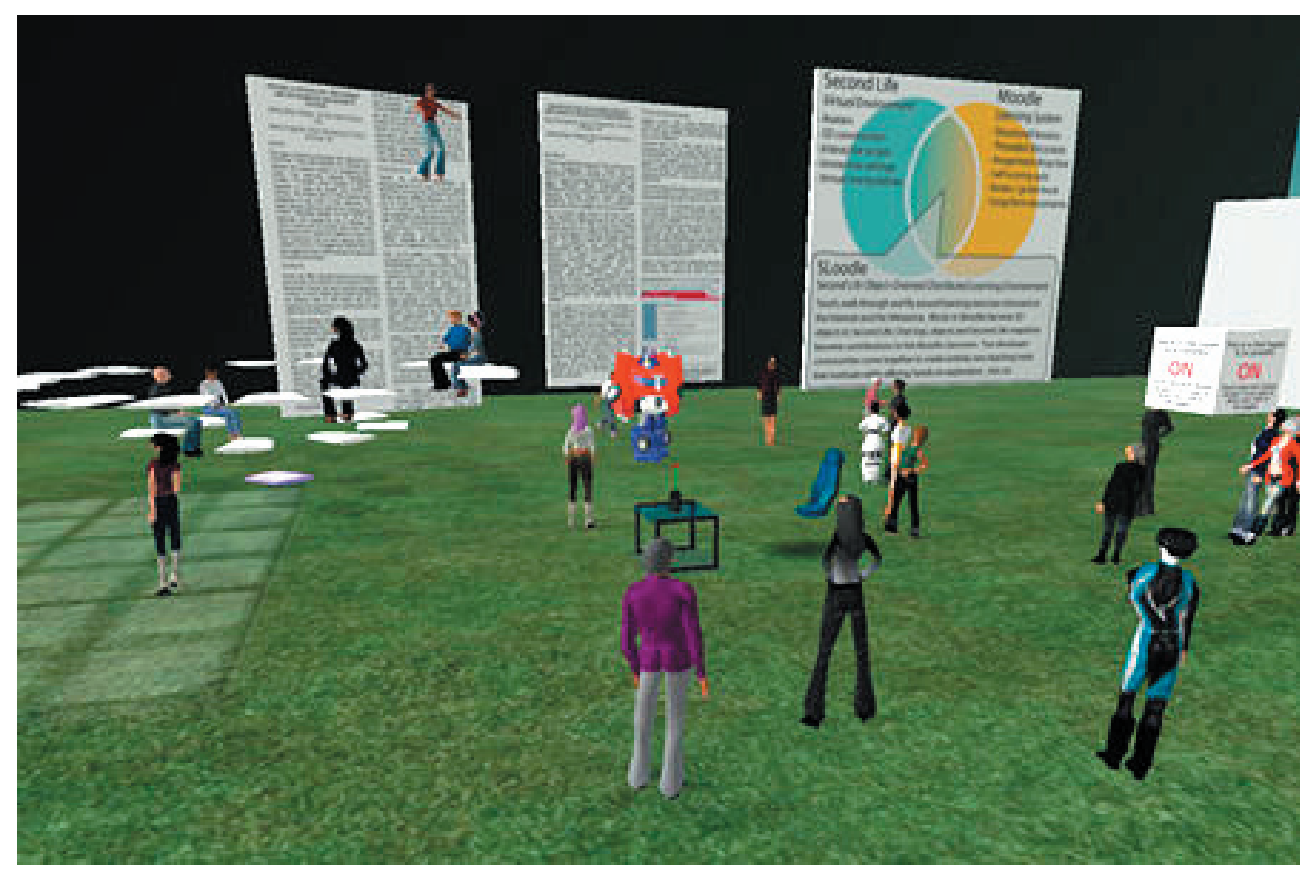

Abbildung 2: Integration einer Lernplattform in einer virtuellen 3-D-Umgebung (Bildquelle: www.sloodle.com)

\section{Interaktivität / Adaptivität}

Die Kategorie der Interaktivität bzw. Adaptivität wurde in die Debatte aufgenommen, als damit begonnen wurde, Lernsysteme in einer Weise zu gestalten, die dem Benutzer eigene Gestaltungsmöglichkeiten einräumte. Während klassische Drill-and-Practice-Programme allenfalls bei der Wahl des Lernweges gewisse Variationen erlaubten (vgl. Meschenmoser 2002: 97), erlauben offene Simulationen (ebd.: 95f) eine tiefer greifende Gestaltung der Umgebung, die aber immer in dem Masse eingeschränkt ist, in dem Programmierer bestimmte Reaktionen auf bestimmte Eingaben vorsehen. In Lernplattformen sind die 
Gestaltungsmöglichkeiten oft zum Teil durch die Kursdesigner festgelegt, von der Funktionalität her ähneln sie den Möglichkeiten, die allgemein bei Webportalen als «Personalisierung» bezeichnet werden, erlauben also die Auswahl und Anordnung bestimmter Informationsangebote innerhalb einer Oberfläche. Seit Mitte der 1990er Jahre gibt es mit Wikis für reguläre Benutzer die Möglichkeit, auch auf die Inhalte von Webseiten umfassend Einfluss zu nehmen, weitere Formen sozialer Software wie Blogs und Podcasts (vgl. Cych 2006: 35f) werden inzwischen in Lernplattformen integriert. Neu ist nun, dass die Software weniger selbst als Interaktionspartner in Erscheinung tritt und stärker die Funktion übernimmt, durch Algorithmen eine oft auch indirekte Interaktion zwischen den Benutzern zu organisieren. In virtuellen 3-D-Umgebungen gehen die Gestaltungsmöglichkeiten hierüber hinaus, indem die sichtbare Umgebung dauerhaft verändert werden kann. Neben Daten in Form von Text-, Audio- und Videodateien lassen sich hier Objekte schaffen, die bestimmte Funktionen innerhalb der virtuellen Welt haben können und unmittelbar mit den Merkmalen entsprechender Objekte aus der Wirklichkeit korrespondieren. Im Sinne der Zeichentheorie von Peirce (vgl. Schäfer 2005: 63 ff) besteht also stärker die Möglichkeit, ikonische Zeichen zur Kommunikation zu nutzen. Während man beispielsweise in textbasierten Kommunikationsmedien einen informellen Begegnungsraum als Bar bezeichnen musste, um seine Funktion zu bezeichnen (vgl. Pätzold 2003: 113), kann eine Bar in einer 3-D-Umgebung einfach daran erkannt werden, dass sie wie eine Bar aussieht. Richtet ein Spieler eine Bar ein, kann sie darüber hinaus sogar zum Teil Funktionen einer solchen erfüllen (man kann sich dort nach den Avataren anderer Mitspieler umschauen, verabreden, Musik hören usw.). Damit erfährt allerdings auch das Konzept der Adaption eine Erweiterung. Ging es bisher vor allem darum, eine (Lern-)Umgebung an die kognitiven Bedürfnisse eines Lernenden anzupassen (Schrittfolge des Lernweges, Ausmass und Art der Rückmeldungen und Hilfen usw.) sowie grundlegende Designvorstellungen zu verwirklichen (Seitenaufbau, Schriftgrösse usw.), so kann eine 3-D-Umgebung zum Teil Funktionen einer realen Umgebung übernehmen und wird damit zum Teil der Lebenswelt der Nutzer. Der soziale Raum, der sich dabei durch computervermittelte Kommunikation konstituiert, gewinnt andere Qualitäten, als dies etwa bei Chats oder innerhalb von Lernplattformen der Fall ist. Er muss nicht nur in kognitiver Hinsicht adaptiv sein, sondern auch emotionalen Bedürfnissen des Lernenden entsprechen können, was für Reinmann bedeutet: Er muss Neugier wecken, Herausforderungen für die eigene Kompetenzentwicklung bieten und die Lernenden müssen in dem Lernraum Vertrauen aufbauen können (vgl. Reinmann 2006: 41). Gelingt dies allerdings, so tritt auch die Bedeutung realer Begegnungen, die bei der gegenwärtigen Thematisierung des Blended Learning deutlich betont wird (vgl. elearingeuropa.info 2007), vermutlich wieder zurück. 


\section{Vernetzungsaspekte}

Virtuelle 3-D-Umgebungen bieten in der Regel alle Möglichkeiten bisheriger virtueller Interaktionsräume, gehen aber noch darüber hinaus. Da sie als Echtzeitumgebungen konzipiert sind, dominiert synchrone Kommunikation, die in der Regel sowohl öffentlich als auch privat erfolgen kann. Öffentlich bedeutet hierbei, dass alle Nutzer in der näheren «Umgebung» das getippte als Sprechblase oder ähnliches sehen können, während private Kommunikation jeweils nur bei dem Adressaten auf dem Bildschirm erscheint. In dieser Hinsicht gleichen die virtuellen 3-D-Umgebungen also Chat-Programmen wie Skype oder IRC. Durch die Herstellung eigener Objekte ist aber auch eine asynchrone Kommunikation möglich, etwa indem Briefe oder Plakate erzeugt werden. Die oben angeführte Schnittstelle Smoodle versucht, gerade in diesem Bereich die Möglichkeiten in Richtung traditioneller E-Learning-Szenarien zu erweitern.

Der Kontakt zu anderen Benutzern lässt sich - ebenfalls analog zu gängigen Chat-Programmen - leicht aufbauen, wenn der Name des Adressaten bekannt ist. Dann kann dieser gesucht und der eigene Avatar in seine Nähe gebracht werden. Ebenso wie in MUDs können aber auch Fremde einfach «angesprochen» werden; in virtuellen 3-D-Umgebungen ist die Hemmschwelle hierzu erfahrungsgemäss recht gering. Die Präsenz von «Unterstützungspersonal» (sowohl in Form von Menschen als auch durch entsprechende Programme) führt zusätzlich zu einer «Kultur des Angesprochen-Werdens», die die Kontaktaufnahme erleichtert.

Ebenso vielfältig sind die Möglichkeiten der Gruppenbildung, wenngleich sie von Software zu Software variieren: In Umgebungen wie Second Life oder Active Worlds können Gruppen sich von selbst räumlich definieren, indem mehrere Avatare dicht beieinander stehen (z. B. vor einem Exponat in einem virtuellen Museum). Daneben kann auch die Gestaltung eines Orts dazu führen, dass sich hier Gruppen bilden (etwa das angesprochene Beispiel einer Bar) und schliesslich kann die Gruppenbildung auch gezielt annonciert werden, z. B. durch Plakate. Im dezentralen Netzwerk Moove basiert die Gruppenbildung auf Einladungen, hier spielt die zusätzliche Webseite des Betreibers ausserhalb der Umgebung eine wichtige Rolle.

\section{Soziale Räume}

Eng mit den Vernetzungsaspekten verbunden ist die Frage nach den Möglichkeiten der Gestaltung sozialer Räume. Es geht hier also nicht mehr nur um die konkreten Kommunikationsmöglichkeiten, sondern um die Möglichkeiten der Gestaltung einer Umgebung, die Kommunikation und Interaktion - und damit soziales Handeln - wahrscheinlich macht. Hier liegt zweifellos ein Schwerpunkt der Möglichkeiten virtueller 3-D-Umgebungen. Schon bei Chat-ähnlichen Diskussionen ist durch die optische Repräsentation der Akteure eine deutlich kla- 
rere Struktur gegeben und das Problem, mehrere Diskussionsstränge parallel zu verfolgen (vgl. Lermen und Pätzold 2004: 42f) ist verringert. In 3-D-Umgebungen können die Akteure zusätzlich durch die Körperhaltung signalisieren, an wen sie sich wenden. Darüber hinaus bestehen aber noch weitere Möglichkeiten, sozialen Nahraum zu strukturieren, etwa indem sich optisch Gruppen bilden, die sich einem bestimmten Thema zuwenden (gleichzeitig erlauben die Programmoberflächen dem Benutzer dennoch, andere Diskussionen weiter zu verfolgen). Betrachtet man beispielsweise Abbildung 2, so gewinnt man bereits aus der Haltung der Avatare mit einem Blick eine komplexe Vorstellung davon, wer sich welchem Thema oder welcher anderen Figur zuwendet. Wie angesprochen können sich die Akteure darüber hinaus durch Gestik und Mimik mitteilen. Bestimmte nonverbale Gesten wie Winken und Umarmen sowie ein mimisches Repertoire erlauben eine emotional getönte Kommunikation. Zur nonverbalen Kommunikation gehört auch die Möglichkeit, nicht zu handeln, also etwa auf Ansprache nicht zu reagieren. ${ }^{9}$ Durch die optische Präsenz des Avatars wirkt das Ausbleiben einer Reaktion sehr viel direkter als etwa bei einem Chat.

Schliesslich beeinflusst die individuelle Gestaltung der Avatare wie auch der Umgebung die Möglichkeiten sozialer Interaktion. Benutzer können sich zu ähnlich aussehenden Figuren hingezogen fühlen, sie können hoffen, über die gemeinsame Vorliebe für eine bestimmte optische Umgebung, eine bestimmte Hintergrundmusik oder Ähnliches leichter Zugang zu anderen Akteuren zu finden, und sie lassen sich natürlich nicht selten von der optischen Repräsentation des Geschlechts bei der Wahl ihrer Kommunikationspartner leiten.

\section{Gemeinschaft}

Gemeinschaft entsteht, wenn in einem sozialen Interaktionsraum gemeinschaftsbildende Handlungen vollzogen werden, etwa ein Regelwerk aufgestellt wird, bestimmte Kommunikationsmöglichkeiten und Formen der Selbstdarstellung eröffnet und genutzt werden usw. (vgl. Marotzki 2003: 156ff). Systemtheoretisch gesprochen lassen sich diese auf die kontinuierliche Herstellung einer Aussengrenze reduzieren (Luhmann 1984: 35), wobei es nicht darum geht, im bildhaften Sinne «auszugrenzen», sondern darum, kontinuierlich die Frage zu beantworten, wer zu einer Gemeinschaft dazugehört und wer nicht. Dies geschieht in realen Gruppen ebenso durch informelle Handlungen, z. B. das Tragen bestimmter Kleidung, wie durch formale Handlungen, z. B. das Eingehen einer Mitgliedschaft. Beide Möglichkeiten sind auch für virtuelle 3-D-Umgebungen beispielhaft. Auch hier lässt sich durch Kleider, Accessoires und Ähnliches Zugehörigkeit zur Schau tragen und ebenso sind formale Mitgliedschaften und durch Passwörter gesicherte Sphären möglich. Während bereits Instant-Messenger und Chat-Programme die Möglichkeit bieten, durch selbst gewählte Bilder zur eigenen Person «etwas darzustellen» und private "Gruppenräume» zu 
öffnen, sind die Möglichkeiten in virtuellen 3-D-Umgebungen potenziert - mit allen positiven und negativen Konsequenzen. Hier kann man durch die Wahl einer entsprechenden Erscheinungsform wichtige soziale Hinweisreize geben, aber es ist auch denkbar, dass käuflich zu erwerbende Ausstattungsmerkmale zu einer sozialen Differenzierung nach ökonomischen Kriterien führen, die in Lernkontexten oft gerade nicht gewünscht ist. Die individuelle Ausstattung der Erscheinungsform (und damit die "Formulierung» einer unmittelbaren, optischen Botschaft) erlaubt unabhängig davon aber, zusammen mit der Raummetapher der 3-D-Welten, neue Formen der Bildung und Aufrechterhaltung virtueller Gemeinschaften. Schliesslich besteht auch die Möglichkeit, durch eher verborgene Hinweise (z. B. bestimmte Gesten) die Zugehörigkeit zu bestimmten Gruppen zu kommunizieren.

Die Dauerhaftigkeit und die Interaktionstiefe solcher Gruppen hängt von einer Vielfalt von Kriterien ab, von denen viele kaum pädagogisch zu beeinflussen sind. Analog zur Interaktion in der realen Welt überformen Aspekte von Sympathie und Antipathie sonstige Kriterien der Zusammenarbeit (wie etwa ähnliche Interessen). Auch die Geschicklichkeit bei der technischen Bedienung der 3-D-Umgebung spielt hier eine Rolle. Diese Effekte lassen sich zwar pädagogisch kontrollieren, indem etwa nur bestimmte Interaktionsformen, Kleider und Ähnliches zugelassen werden, sie würden dann aber gerade jene Aspekte der virtuellen 3-D-Umgebung blockieren, die diese gegenüber anderen Interaktionsformen gerade interessant macht.

Die Vergemeinschaftung (und ebenso die Ausgrenzung) in virtuellen 3-D-Umgebungen hat medienpädagogisch weit reichende Konsequenzen. Hipfl und Hug stellen, im Anschluss an Zygmund Baumann, dar, dass Gemeinschaften eine Leerstelle in der Alltagserfahrung vieler Menschen füllen, dass man durch Gemeinschaft der ansonsten allgegenwärtigen Erfahrung begegnen kann, «ganz alleine mit den Problemen des Alltags zurechtkommen zu müssen, diese mit niemandem teilen zu können, es mit unerwarteten und unbekannten Dingen zu tun zu haben, mit Menschen in Kontakt zu kommen, die man nicht kennt und bei denen man nicht sicher ist, dass man ihnen vertrauen kann» (Hug und Hipfl 2006: 2). Gleichzeitig geht aber auch von der Begegnung mit Unbekanntem und Unbekannten ein Reiz aus (vgl. Turkle 2006: 6), der im Zeitalter lebenslangen Lernens geradezu konstitutiv erscheint. Interessanterweise bieten virtuelle 3-D-Umgebungen mitunter die Möglichkeit, beide Erfahrungen, die der Gemeinschaft und die der Begegnung mit Unbekannten, miteinander zu verbinden. Denn durch die Wahl einer Gemeinschaft, die sich durch visuelle und weitere Hinweise als den eigenen Interessen entsprechend präsentiert, lässt sich eine Gruppe durchweg fremder Akteure finden, bei denen dennoch von einem hohen Ausmass an Interessenkohärenz ausgegangen werden kann, zumindest hinsichtlich des durch Aussehensmerkmale usw. definierten Bereichs. Ebenso eignen sich 
bestimmte Orte dazu, interessensähnliche Gruppen zu konstituieren, ohne dass die Akteure vorher umfassende Skripts des Kennenlernens durchlaufen müssten. Nach Hug und Hipfl beruhen mediale Gemeinschaften auf Vertrautheit und emotionaler Bindung (ebd.: 17) und es liegt auf der Hand, dass optische Repräsentationen genutzt werden können, um Vertrautheit zu fördern, ebenso wie die teilweise emotional getönten Gesten der Avatare Bindungen befördern können. Die detailliertere Auflistung gemeinschaftsstiftender Aspekte (vgl. ebd.; Marotzki 2003: 156ff) passt in erstaunlichem Masse auf die Merkmale virtueller 3-D-Umgebungen. So leisten diese umfassende Möglichkeiten der Differenzierung der raum-zeitlichen Organisation und Präsentation von Individuen in einer Gemeinschaft, können umfassende, regelgeleitete Teilhabemöglichkeiten realisieren und erlauben ein komplexes Zusammenspiel von «Faktizität und Fiktionalität» (ebd.: 18).

So können in virtuellen 3-D-Umgebungen starke gemeinschaftliche Bindungen zwischen Paaren, kleineren und grösseren Gruppen entstehen, die nicht nur auf einem gemeinsamen Interesse oder einer Aufgabe beruhen, sondern mit emotionaler Zuwendung, Sympathie und ähnlichem zu tun haben. Diese Art von Bindung entsteht aber nur dort, wo sie zugelassen wird, was die mediendidaktische Gestaltung von virtuellen Umgebungen in ein Dilemma stürzt. Auf der einen Seite erscheint die Entstehung von Bindungen positiv, weil sie das kooperative Arbeiten, die emotionale Beteiligung und damit auch den Lernerfolg positiv beeinflusst - auf der anderen Seite verlangt sie nach Bedingungen, die durchaus nicht alle unmittelbar dem Lernerfolg zuträglich sind - als wichtigste wäre hier zu nennen, dass die soziale Interaktion ganzheitlich sein muss, sich also nicht allein auf einen fachlichen Diskurs beschränken darf.

\section{Integration}

Für die Gestaltung von Lernumgebungen ist letztlich der Bildungswert des Lernprozesses entscheidend, die Wahrscheinlichkeit also, mit der die Lernenden in der Umgebung tatsächlich eine Weiterentwicklung erfahren. Die medienpädagogische Diskussion hat dabei als Richtschnur «weitgehend das Konzept situierten Lernens anerkannt» (Tulodziecki 2005: 6), bei dem die Lernsituationen in authentischer Weise die Anforderungen der Anwendungssituation widerspiegeln sollen und bei dem sozialer Austausch, Reflexion und mehrperspektivische Sichtweisen wichtige Rollen spielen. Virtuelle 3-D-Umgebungen liefern gute Voraussetzungen für ein solches Lernen: Sie erlauben - wie beschrieben - vielfältige Möglichkeiten sozialer Interaktion, in ihnen lassen sich anspruchsvolle und wirklichkeitsnahe Simulationen entwickeln und sie erlauben vielfältige Zugänge zu einem Sachverhalt. Dennoch legen Theorie und Empirie zwei Einwände nahe, die das Potenzial solcher Umgebungen etwas kritischer erscheinen lassen: Zum einen verlangt eine solche Umgebung - wie bereits angesprochen 
- einen Interaktionsraum, der deutlich mehr Handlungsmöglichkeiten anbietet, als aus unterrichtlicher Sicht gewünscht sein mögen. Zum anderen ist die Frage nach «Wirklichkeitsnähe» unter Berücksichtigung der Modelle virtueller Gruppenbildung und virtueller Identität prekär. So weisen insbesondere Turkles Arbeiten zur multiplen Identität darauf hin, dass eine im virtuellen Raum gemachte Erfahrung durchaus nicht selbstverständlich auf das Handeln im realen Leben zurückwirkt. Akteure in virtuellen Realitäten verfügen nach Turkle zwar über ein integriertes Selbstkonzept, aber sie schaffen es sich, «indem sie durch viele verschiedene Identitäten vagabundieren» (Turkle 1998: 287). Die Merkmale dieser verschiedenen Identitäten, einschliesslich erzieherisch zu beurteilender Aspekte wie Höflichkeit, Aufmerksamkeit, Aggression und Ähnlichem sind nicht automatisch Bestandteil des Selbst, sondern werden gewissermassen auf die Identitäten verteilt. So ist vorstellbar, dass ein Akteur sich in einer simulieren politischen Aktion in einem Rollenspiel in Second Life als verantwortungsbewusster und besonnener Friedensaktivist erweist, ohne eine auch nur ähnliche Position in anderen (virtuellen oder realen) Kontexten zu vertreten. ${ }^{10}$ Insofern gewinnt der ohnehin komplexe Zusammenhang zwischen Lern- und Anwendungsfeld im Rahmen des Transfers (vgl. Hof und Lambert 2004) in virtuellen 3-D-Umgebungen eine zusätzliche Dimension: Wie realistisch ist die Annahme, dass in einer virtuellen Umgebung (und damit im Extremfeld einer virtuellen Identität) gewonnene Erfahrungen, Einsichten und Kompetenzen auch in anderen Umgebungen (und Identitäten) wirksam werden?

\section{Weitere Perspektiven}

Für das pädagogische Potenzial virtueller 3-D-Umgebungen ist neben den genannten Merkmalen natürlich auch die technische Umsetzung von Bedeutung. Bereits angesprochen wurden die zum Teil erheblichen Anforderungen an die Endgeräte der Benutzer. So erfährt etwa die Bildung von (Lern-)Gemeinschaften eine faktische Einschränkung in der Begrenzung potenzieller Mitglieder auf solche Personen, die einen hochwertigen Rechner mit schnellem Netzzugang regelmässig zur Verfügung haben. Auch ist die Bedienung der verschiedenen Programme, die den Zugang in virtuelle Umgebungen regeln, nicht immer unproblematisch. Insbesondere die Software von Second Life wird in technisch orientierten Diskussionsforen und Blogs mit einer eigentümlichen Mischung aus Bewunderung und süffisantem Hohn kommentiert, weil sie einerseits ein bis vor kurzem kaum vorstellbares multimediales Netzwerk realisiert, andererseits aber auch vier Jahre nach dem Start noch nicht einigermassen zuverlässig lauffähig ist, sondern immer noch in verschiedensten Situationen den Dienst quittiert. Hinzu kommt, dass gegenwärtig weite Teile virtueller 3-D-Welten nur nutzbar sind, wenn man Englisch versteht - wie in der Anfangszeit des WWW sind ande- 
re europäische Sprachen hier noch vergleichsweise wenig vertreten, wenngleich der Umfang zunimmt.

Etwas weiter in die Zukunft gedacht stellt sich die Frage, ob die Entwicklung virtueller 3-D-Umgebungen nicht ohnehin mit dem Niedergang des PCs als Endgerät der Wahl kollidiert (vgl. Anderson 2006). Schon heute zeichnet sich ab, dass wesentliche Aspekte der Informations- und Kommunikationstechnologien auf mobile Endgeräte abgebildet werden müssen, wenn sie dauerhaft Bestand haben sollen (vgl. Sharples 2006: 21; Gayesky 2002)11.

\section{Fazit}

Die bisherigen Ergebnisse zu virtuellen 3-D-Umgebungen zeigen zweierlei: Zum einen handelt es sich zweifellos um technisch ambitionierte Erweiterungen der bisherigen Möglichkeiten computervermittelter Kommunikation und Gemeinschaftsbildung, die auch ein medienpädagogisches Potenzial haben. Gleichzeitig ist das Potenzial in Bezug auf den Einsatz solcher Umgebungen als Lernwelten aber bisher auf recht spezielle Nischenanwendungen beschränkt. Im Wesentlichen liegt es in folgenden pädagogischen Aufgaben:

- Bildungsmarketing: Virtuelle 3-D-Umgebungen bieten die Möglichkeit, gezielt spezielle Zielgruppen anzusprechen. In dem Masse, in dem Bildungsanbieter auf einem konkurrenzökonomischen Markt konkurrieren, können sie (noch) damit rechnen, durch eine Präsenz in Second Life und ähnliche Umgebungen einen zusätzlichen Publicity-Mehrwert zu erreichen.

- Produktentwicklung und -erprobung: Ebenso wie andere Dienstleistungen lassen sich auch Bildungsangebote in einer virtuellen Umgebung erproben. Zwar existieren bisher keine Standardangaben zur Übertragbarkeit von Erfahrungen aus virtuellen Umgebungen in die Realität, dennoch eignet sich die virtuelle Umgebung mitunter für Machbarkeitsstudien, die sich dann auch in anderen Umgebungen umsetzen lassen.

- Entwicklung spezieller virtueller Angebote: Innerhalb verschiedener Umgebungen können Kunden eigene Lernangebote entwickeln und implementieren, seien es virtuelle Hörsäle, Ausstellungen oder anderes. Besonders populär scheint dabei die Nutzung der 3-D-Technik zur Konstruktion von anschaulichen Simulationen, etwa im Bereich der Naturwissenschaft. Bestimmte Anbieter wie Active Worlds bieten darüber hinaus die Möglichkeit, auf Grundlage der hier verwendeten Softwarewerkzeuge eigene, abgeschlossene (Lern-)Umgebungen zu kreieren, die nicht mit der öffentlichen Netzinfrastruktur verbunden sein müssen. Gänzlich unerschlossen ist demgegenüber die Möglichkeit, soziale Prozesse zu simulieren. Die Möglichkeiten klassischer Planspiele (Rebmann 2001) erscheinen in virtuellen Umgebungen potenziert und unter Berücksichtigung ethischer Standards wären komplexe und tiefgreifende Simulationen sozialer Vorgänge wie Exklusion und Inklusion und 
anderes denkbar, die sich in realen Settings ungleich schwerer abbilden liessen.

- Sozialwissenschaftliche Erkundung: Die «Communities» in Second Life, Active Worlds und anderen virtuellen Umgebungen agieren in vielerlei Hinsicht als soziale Gemeinschaften, die entsprechende sozialwissenschaftlich relevante Merkmale aufweisen. So lassen sich im Rahmen von virtuellen Umgebungen Fragestellungen wie die Bildung und Auflösung von Gruppen, die Entstehung von Skripts und Ähnliches gewissermassen unter Laborbedingungen (vgl. Turkle 1998: 289) beobachten. Zwar muss klar sein, dass die Gruppenbildung in einer solchen Umgebung nicht unbedingt in jedem Punkt mit der Bildung realer Gruppen vergleichbar ist, dennoch werden charakteristische Merkmale sozialer Prozesse sichtbar, teilweise sogar deutlicher als im realen Leben. Gleichzeitig erlaubt die Beobachtung in virtuellen Umgebungen eine einfache Dokumentation (in manchen Oberflächen ist auch das automatische Erstellen von «Videos» möglich) und ist im Allgemeinen weniger invasiv, weil unbeteiligte Beobachter hier (noch) einen relativ normalen Bestandteil der Umwelt darstellen. ${ }^{12}$

- Neben den angesprochenen mediendidaktischen Fragen stellen sich natürlich auch hier wieder Fragen zur Medienerziehung, etwa zur Wirkung der «virtuellen Vergemeinschaftung» auf reale soziale Beziehungen, zum Jugendschutz und Ähnliches. Auch wenn diese in anderen Kontexten bereits beantwortet worden sind, gewinnen sie hier zum Teil einen neuen Charakter, weil sie mit Interaktionsmöglichkeiten verbunden sind, die bei anderen Medien so nicht vorhanden sind.

Wie bei anderen Medien auch wird die Begeisterung für virtuelle 3-D-Umgebungen vermutlich in der nächsten Zeit einer gewissen Ernüchterung weichen. Die Medienpädagogik verhält sich - zumindest in der deutschsprachigen Diskussion - auffällig zurückhaltend gegenüber den neuen Möglichkeiten, die hier entstehen. Daran dürften auch die relativ hohen Kosten mit Schuld sein, die durch die Mieten von "Land» in entsprechenden Umgebungen sowie die Entwicklung der notwendigen Programmierfähigkeiten entstehen. Allerdings sind gerade die ökonomischen Bedingungen der virtuellen Umgebungen sicher noch einem starken Wandel unterworfen und ähnlich wie bei der Webseitengestaltung ist zu erwarten, dass mittelfristig Software angeboten wird, die auch die Gestaltung in virtuellen 3-D-Welten erheblich vereinfacht. Insofern erscheint das Web 3.0, wie es in Anspielung auf die dritte Dimension mitunter genannt wird, noch nicht als alltagstaugliche Lernumgebung, es ist aber absehbar, dass es mittelfristig das mediendidaktische Handlungsrepertoire erweitern wird und dass diese Erweiterung - im Gegensatz zu Entwicklungen im Bereich der ersten Generationen des E-Learning - über elementare Formen des Visualisierens und Präsentierens weit 
hinausgehen werden. Die Erprobung und Evaluation didaktischer Settings in virtuellen 3-D-Umgebungen, die Untersuchung ihrer Auswirkungen hinsichtlich der Interaktion in der 3-D-Umgebung, zwischen virtuellem und realem Raum sowie der Umgang mit den virtuellen Lernerfahrungen ausserhalb der Lernumgebung (vgl. Marotzki 2003: 151ff) stellen dann zunehmend wichtige medienpädagogische Aufgaben dar.

\section{Fussnoten}

1 Massive Multiplayer Online Role-Playing Game, also Spielszenarien, bei denen die Akteure in Rollen schlüpfen, miteinander online und in Echtzeit interagieren und gleichzeitig sehr viele Spieler aktiv sein können.

2 Bühl geht wie viele andere davon aus, dass zum Eintauchen, der «lmmersion» in eine virtuelle Welt, Techniken wie Datenhandschuh und Datenhelm unabdingbar wären. Nimmt man als Indikator für die Immersion etwa Flow-Erleben (vgl. Reinmann 2006: 41), so lässt sich leicht zeigen, dass gerade dies nicht der Fall ist. Die Untersuchungen Turkles seit den 1980er Jahren beispielsweise belegen einen hohen Grad an Immersion sogar in textbasierten Interaktionsumgebungen (vgl. Turkle 1998).

3 Die Abkürzung MUD für zumeist textbasierte Interaktionsumgebungen für grosse Zahlen vernetzter Anwender wird wahlweise mit «Multi User Dungeon» oder «Multi User Domain» übersetzt. Erstere Version knüpft an die ursprüngliche Idee an, ein Fantasy-Rollenspiel («Dungeons and Dragons») als Netzwerkspiel zu implementieren, letztere verweist darauf, dass diese Art von Spielhandlung inzwischen nicht mehr essenziell für solche Systeme ist (vgl. Turkle 1998).

4 In einer Umgebung, die den ersten beiden Punkten genügt, ist immer auch eine Spielhandlung möglich - gerade die Feststellung, dass dies der Fall ist, kann als Heuristik dafür dienen, ob die Umgebung auch sonst anspruchsvolle Interaktionen erlaubt - «könnte man hier auch ein Rollenspiel spielen?».

5 In Anspielung auf die Plattform Second Life wird diese manchmal ironisch «first life» genannt, vgl. www.getafirstlife.com [26.3.2007].

6 Die volkswirtschaftliche Einordnung einer solchen "Spielwährung» ist nicht ganz einfach (vgl. Hamann/Uehlecke 2007), für die weiteren Überlegungen aber auch nicht zentral.

${ }^{7}$ Beispielsweise kleines «Areal» der Univeristätsbibliothek Konstanz oder eine Umgebung, die die Zeit van Goghs darstellen soll und in der grafischen Anmutung dessen Bildern nachempfunden ist.

${ }^{8}$ Bei Rechnern, die im Bildungsbereich eingesetzt werden, also nicht auf Spielanwendungen hin optimiert sind, wird die Grafikkarte diesen Anforderungen oft nicht gerecht, was zu einem relativ langsamen und nicht störungsfreien Bildaufbau führt. Auch andere 3-D-Umgebungen profitieren in der Regel von einer entsprechenden Grafikhardware, sind aber nicht so abhängig davon. 
9 Ein wichtiges Detail bei Second Life mag dabei sein, dass man - im Gegensatz zu chats - sieht, wenn das Gegenüber anfängt, eine Antwort zu tippen. Zwar wird nicht die Antwort selbst gezeigt, aber der Avatar vollführt Bewegungen, als tippe er auf einer unsichtbaren Tastatur. So ist bereits vor einer Antwort ersichtlich, ob eine solche zu erwarten ist.

${ }^{10}$ Die Konsequenzen hiervon reichen weit über das Feld der Mediendidaktik hinaus. So geht man davon aus, dass transformatives Lernen (vgl. Mezirow 1997) oder Identitätslernen (Siebert 2003: 84) als zentrale Prozesse des Lernens im Lebenslauf identitätsverändernde Formen sind, mit denen schwerwiegenden Verunsicherungen begegnet wird. Werden diese Verunsicherungen in virtuellen Umgebungen bearbeitet, so wäre denkbar, dass allenfalls «ein Teil der Identität» bzw. nach Turkle ein Teil des Selbst an dieser Veränderung teilhat.

${ }^{11}$ Es gibt bereits Versuche, Second Life auf Handys zu portieren (http://secondlife.reuters.com/stories/2007/02/08/comverse-demos-second-lifeon-mobile-phones [27.3.2007]). Dabei ist aber noch unklar, welche Funktionalität auf normalen Endgeräten tatsächlich erreicht werden kann.

${ }^{12} \mathrm{Da}$ man es bei den Akteuren letztlich mit Personen zu tun hat, stellen sich auch hier mittelfristig forschungsethische Fragen, die aber an dieser Stelle noch nicht diskutiert werden sollen.

\section{Literatur}

Anderson, Paul. «The Future of Human Computer Interaction.» Emerging Technologies for Learning. Hrsg. v. BECTA. Coventry, UK: BECTA, 2006. 24-31.

Bloh, Egon; Lehmann, Burkhard. «Online-Pädagogik - der dritte Weg? Präliminarien zur neuen Domäne der Online-(Lehr-)Lernnetzwerke (OLN).» Online-Pädagogik. Hrsg. v. Burkhard Lehmann und Egon Bloh. Baltmannsweiler: SchneiderVerlag Hohengehren, 2001. 11-128.

Bühl, Achim. Die virtuelle Gesellschaft des 21. Jahrhunderts: Sozialer Wandel im digitalen Zeitalter. 2. Aufl. Wiesbaden: Westdeutscher Verlag, 2000.

Doherty, Paul; Rothfarb, Rob; Barker, Dave. «Building an Interactive Science Museum in Second Life.» Proceedings of the Second Life Education Workshop at the Second Life Community Convention San Francisco August 20th, 2006. Hrsg. v. Daniel Livingstone u. Jeremy Kemp. Paisley, UK: University of Paisley, 2006. 19-24.

Cych, Leon. «Social Networks.» Emerging Technologies for Learning. Hrsg. v. BECTA. Coventry, UK: BECTA, 2006. 32-41.

elearningeuropa.info. eLearning Papers 3: Blended Learning. «http:// www.elearningpapers.eu/index.php?page=volume_pdf_downloads (28.3.2007).

Forrai, Gábor. «The Epistomology of the Hypertext.» Mobile Learning: Essays on Philosophy, Psychology and Education. Hrsg. v. Kristóf Nyíri. Wien: Passagen, 2003. 35-45.

Gayeski, Diane. LearningUnplugged: Using Mobile Technologies for Organizational Training and Performance Improvement. New York: AMACOM, 2002. 
Hamann, Götz; Uehlecke, Jens. «Die nächste Kolonie des Kapitalismus.» Die Zeit, Nr. 2., 4.1. 2007: 17-18. Online unter: http://www.zeit.de/2007/02/ Second-Life

Hof, Christiane; Lambert, Astrid. «Lernerwartung und Lerntransfer: Anmerkungen zum Transfer von Lernergebnissen in der Allgemeinen Erwachsenenbildung.» Lernen und Handeln: Eine Grundfrage der Erwachsenenbildung. Hrsg. v. Walter Bender, Maritta Gross und Helga Heglmeier. Schwalbach / Ts.: Wochenschau Verlag, 2004. S. 197-212.

Hug, Theo; Hipfl, Brigitte. «Medien-Gemeinschaften: Aktuelle Diskurse und konzeptionelle Analyse.» In: MedienPädagogik 26.4.2006: www. medienpaed.com/2006/hug-hipfl0604.pdf.

Marotzki, Winfried. "Online-Ethnographie - Wege und Ergebnisse zur Forschung im Kulturraum Internet.» Jahrbuch Medienpädagogik 3. Hrsg. v. Ben Bachmair. Opladen: Leske + Budrich, 2003. 149-165.

Meschenmoser, Helmut. Lernen mit Multimedia und Internet. Baltmannsweiler: Schneider Verlag Hohengehren, 2002.

Lermen, Markus; Pätzold, Henning. "Chats als Lernräume in der Hochschulausbildung.» merz 1 (2004): 40-45.

Livingstone, Daniel; Kemp, Jeremy (Hrsg.). Proceedings of the Second Life Education Workshop at the Second Life Community Convention San Francisco August 20th, 2006. Paisley, UK: University of Paisley, 2006.

Luhmann, Niklas. Soziale Systeme: Grundriss einer allgemeinen Theorie. Frankfurt/Main: Suhrkamp, 1984.

Pätzold, Henning: «Opening Gates - virtuelle Konferenzen als Möglichkeit des internationalen pädagogischen Austauschs.» Tertium Comparationis 2 (2003): 111-123.

Peterson, Donald. "Context and the E-Condition.» Mobile Learning. Essays on Philosophy, Psychology and Education. Hrsg. v. Kristóf Nyíri. Wien: Passagen, 2003. 117-125.

Schäfer, Karl-Hermann. Kommunikation und Interaktion: Grundbegriffe einer Pädagogik des Pragmatismus. Wiesbaden: VS Verlag, 2005.

Rebmann, Karin. Planspiel und Planspieleinsatz: Theoretische und empirische Explorationen zu einer konstruktivistischen Planspieldidaktik. Hamburg: Dr. Kova, 2001.

Reinmann, Gabi. «Ist E-learning eine pädagogische Innovation? Anregungen für eine Förderung von Lernkultur und Emotionen in unseren Bildungsinstitutionen.» eLearning-Didaktik. Hrsg. v. Rolf Arnold u. Markus Lermen. Baltmannsweiler: Schneider Verlag Hohengehren, 2006. $31-46$.

Sharples, Mike. "How can we adress the conflicts between personal informal learning and traditional classroom education?» Big Issues in Mobile Learning. Hrsg. v. Mike Sharples. Nottingham, UK: University of Nottingham, 2006. 21-24.

Tulodziecki, Gerhard. «Zur Situation der Medienpädagogik in der Bundesrepublik Deutschland.»In: MedienPädagogik 5.10.2005: www.medienpaed.com/05-1/tulodziecki05-1.pdf.

Tulodziecki, Gehard und Herzig, Bardo. Mediendidaktik. Stuttgart: Klett, 2004. 
Turkle, Sherry. Leben im Netz: Identität im Zeitalter des Internet. Reinbek b. Hamburg: Rowohlt, 1998.

Turkle, Sherry. "Always-on/Always-on-you: The Tethered Self.» Erscheint in Handbook of Mobile Communication and Social Change. Hrsg. v. James Katz. Cambridge, MA: MIT Press. Vorabveröffentlichung unter 〈http://web.mit.edu/sturkle/www/Always-on\%20Always-on-you_ The\%20Tethered\%20Self_ST.pdf> (18.7.07).

Vollbrecht, Ralf. Einführung in die Medienpädagogik. Weinheim und Basel: Beltz, 2001. 